\title{
Acute limb ischemia due to intracardiac myxoma in a patient with atrial fibrillation
}

\author{
Anna Szymanska ${ }^{1}$, Joanna Syska-Suminska ${ }^{1}$, Jerzy Rekosz ${ }^{1}$, \\ Anna Skrobisz ${ }^{1}$, Anna E. Platek ${ }^{2}$, Miroslaw Dluzniewski ${ }^{1}$ \\ ${ }^{1}$ Department of Heart Diseases, The Medial Center of Postgraduate Education, Warsaw, Poland \\ ${ }^{2}$ Department of General and Experimental Pathology with Center for Preclinical Research \\ and Technology (CEPT), Medical University of Warsaw, Poland
}

An 82-year-old female with a history of paroxysmal atrial fibrillation was hospitalized due to exertional dyspnea which had lasted for 2 weeks, with no peripheral edemas. The transthoracic echocardiography (TTE) revealed a mass in the left atrium $(1.6 \times 2.2 \mathrm{~cm})$ with connective tissue peduncle attached in the area of fossa ovalis in the intraarterial septum (Fig. 1A, B). The transesophageal echocardiography (TEE) confirmed mobile mass in the left atrium suggesting atrial myxoma (Fig. 1C). Cardiac myxoma removal was offered to the patient, but the patient did not agree to the procedure and was discharged.

Twenty hours after the discharge, the patient presented again in the Emergency Room due to pulmonary edema in the course of rapid atrial fibrillation and symptoms of acute left limb ischemia (no femoral arterial pulse, coldness, cyanosis, and paralysis). TTE showed no myxoma mass in the left atrium. Only connective tissue peduncle remained in the area of fossa ovalis in the intraarterial sep- tum (Fig. 1D, E). Vascular ultrasonography was performed and demonstrated complete occlusion of the left femoral artery. The mass was recovered via percutaneous intervention and examination confirmed it to be myxoma. Revascularization was provided immediately and ensured no vascular or neurological defects. Subsequent TEE revealed a small residue of connective tissue peduncle in the area of fossa ovalis (Fig. 1F). The patient was discharged without complications.

Myxomas can manifest in miscellaneous ways. This might include a fever of unknown origin, acute myocardial infarction, stroke, paraneoplastic effects (including vasculitis, hematological changes, constitutional symptoms). While ischemic symptoms are relatively frequent, they are more often associated with a blood clot mobilization. Tumor embolization in peripheral limb circulation is extremely rare. Nevertheless, myxomas must be kept in mind as one of the probable causes of thromboembolic complications in various vascular sites.

Conflict of interest: None declared

Address for correspondence: Miroslaw Dluzniewski, MD, PhD, Medical Center of Postgraduate Education, ul. Poznańska 22, 00-685 Warszawa, Poland, tel: +48 2252512 63, e-mail: miroslaw.dluzniewski@cmkp.edu.pl

Received: 26.06.2019

Accepted: 1.10.2019 




Figure 1. The echocardiography studies; A. Transthoracic echocardiography (TTE) on admission — parasternal long axis view; B. Transesophageal echocardiography (TEE) on admission - 4-chamber apical view; C. TEE on admission; D. TTE after vascular intervention — parasternal long axis view; E. TTE after vascular intervention — 4-chamber apical view; F. TEE after vascular intervention. 